\title{
Plastic Effect on the Sliding Inception Between a Cylinder and a Rigid Flat
}

\author{
S. Zhang ${ }^{1,4} \quad$ J. Huan ${ }^{1,4} \quad$ H. Song ${ }^{2} \quad$ X. Liu $^{1 \star} \quad$ Y. G. Wei ${ }^{3 \star}$ \\ $\left({ }^{1}\right.$ LNM, Institute of Mechanics, Chinese Academy of Sciences, Beijing 100190, China) \\ ( ${ }^{2}$ Department of Mechanical Engineering, Johns Hopkins University, Baltimore, MD 21218, USA) \\ $\left({ }^{3}\right.$ College of Engineering, Peking University, Beijing 100871, China) \\ ( ${ }^{4}$ School of Engineering Science, University of Chinese Academy of Sciences, Beijing 100049, China)
}

Received 28 May 2018; revision received 11 September 2018; Accepted 12 September 2018

(c) The Chinese Society of Theoretical and Applied Mechanics 2018

\begin{abstract}
The effects of material plasticity and local slip on the sliding inception of asperity are studied in the present work. Firstly, a semi-analytical solution is derived under the full-stick condition to analyze the effect of material plasticity on sliding friction. Then, a friction model with contact stiffness criterion is proposed to study the cases from partial-slip condition to full-stick condition. Finite element simulations with the provided model are used to present the friction map. The friction coefficient of full-stick interface converges at a stable value, approximately 0.3. Plasticity saturation appears as the normalized contact interference $\omega^{*}$ is larger than 3. A transition mechanism from slip-dominated to yield-dominated takes place in the sliding process. The simulation results are compared with the semi-analytical solution.
\end{abstract}

KEY WORDS Material yielding, Local slip, Contact stiffness criterion, Finite element simulation

\section{Introduction}

Contact and friction are closely related to engineering industry, especially in the technologies associated with rolling bearing, wheel-rail control, head-disk interaction, etc. Understanding the friction law of contacts is critical in the design of engineering systems.

Sliding across the interface is an integration of various factors, including material properties, interfacial roughness, local slip, chemical reaction. The amount of force required for sliding has been a hot topic in the past decades. The classical study of the sliding of a single contact stemmed from Mindlin in 1940s [1]. According to the Mindlin model, the contact patch between two spheres consists of a central stick region surrounded by an annular slip zone. The central stick region gradually diminishes and finally disappears as the tangential load increases, since the material in the central stick region cannot sustain infinite traction. Different from the analytical solution of Mindlin, Hamilton [2] derived an explicit expression for the stress field beneath a sliding contact, which has a non-axisymmetric shape. Further, Chang et al. [3] (CEB friction model) assumed that sliding starts at the instant of yielding, and used the von Mises yield criterion with Hamilton stress field [2] to determine the maximum tangential load of a single asperity contact. Since the first yield point in a spherical contact is surrounded

\footnotetext{
* Corresponding authors. E-mails: xiaomingliu@imech.ac.cn; weiyg@pku.edu.cn
} 
by large volume of elastic material, their results underestimated the real maximum tangential loading that a single junction can support.

Taking material plasticity into account, Kogut and Etsion [4] combined the finite element analysis with an analytical expression to provide a semi-analytical solution for the sliding inception of an elastic-plastic spherical contact. In another study, Brizmer et al. [5] considered the local slip effect and they studied the influences of full stick and perfect slip on elasticity terminus for the first time and then extended the research to the elastic-plastic spherical contact [6]. They concluded that the local slip condition (either full stick or perfect slip) became more dominant on the evolution of the plastic zone with increasing interference. In addition, other models have made attempts to discuss whether the full-stick condition [7] or perfect slip condition [8, 9] should be applied to the realistic interface.

The finite element method (FEM) was employed to study the sliding inception between a deformable sphere (cylinder) and a rigid flat. The sliding friction under highly adhesive conditions was studied by Brizmer et al. [10], and the plasticity effect on friction parameters such as the junction tangential stiffness, static friction force and static friction coefficient was extensively investigated. The full-stick condition was used for the assumption of strong adhesion. In a similar study [11], the adhesive friction of an elastic-plastic cylinder in contact with a rigid flat was investigated, the results of which built up the connection between the static friction and the ductility of the junction. Later on, the full-stick model has been relaxed to the partial-slip condition by Shi and $\mathrm{Wu}[12-14]$ for a better characterization of the friction process. Their proposed model revealed a friction transition between the KE model [4] and the BKE model [10] (full-stick model). However, they did not analyze the competition mechanism between the material plasticity and the local slip effects on sliding initiation.

Most of the work mentioned above either studied the plasticity effect in the full-stick model or the partial slip in the frame work of Mindlin. Few studies paid attention to the transition mechanism from plastic yielding to full slip during sliding inception, and an accurate prediction is still missing. The main goal of this paper is to understand the competition mechanism between the two factors and to provide a quantitative friction map.

In the present work, firstly, a semi-analytical solution for the sliding friction between an elasticperfectly plastic cylinder and a rigid flat is provided. Then, a finite element model for sliding inception is developed by utilizing the contact stiffness criterion, plasticity effect, as well as the local slip effect on static friction. Plasticity versus slip evolution is compared to investigate the sliding transition from full slip to full plastic yielding.

\section{A Semi-Analytical Solution of Sliding Inception Under Full-Stick Condition}

\subsection{Analytical Solution of Friction Coefficient for Elastic Preload}

The friction between a cylinder and a rigid flat is a classical problem in contact mechanics. As an infinitely long cylinder is in contact with the rigid flat, the stress/strain field is assumed to be a $2 \mathrm{D}$ plane strain problem. In the present work, a study of the contact between an elastic-perfectly plastic cylinder (of radius $R$ ) and a rigid flat is shown in Fig. 1. The rigid flat is under the combined vertical load $P$ and horizontal load $Q$. The vertical load $P$ is acted in the $z$-direction, and the horizontal force $Q$ is applied in the $x$-direction in the coordinate system in Fig. 1 . The interference $\omega$ and the contact radius $a$ correspond to the vertical load $P$.

The slip initiates when all surface contact points reach the maximum allowed local shear stress (local yield stress). The normal stress field at the contact surface is determined according to classical contact mechanics (as in Appendix A), so the maximum allowed local shear stress $\tau_{x z}^{*} / Y$ ( $Y$ : yielding stress of the material) can be determined according to the von Mises criterion [15], similar to the previous study [4]. The integration of $\tau_{x z}^{*}$ over the contact patch gives the maximum tangential force $Q_{\max }$, whose value can be used to calculate the friction coefficient.

The pressure distribution by normal load $P$ has the form: $p(x)=p_{0}\left(1-x^{2} / a^{2}\right)^{1 / 2}$, where $p_{0}$ is the maximum contact pressure and $a$ is the contact radius. The equivalent von Mises stress [16] which correlates with the yield stress has the form:

$$
\sigma_{\mathrm{eq}}=\left\{\frac{1}{2}\left[\left(\sigma_{x}-\sigma_{y}\right)^{2}+\left(\sigma_{y}-\sigma_{z}\right)^{2}+\left(\sigma_{z}-\sigma_{x}\right)^{2}+6\left(\tau_{x y}^{2}+\tau_{z y}^{2}+\tau_{z x}^{2}\right)\right]\right\}^{\frac{1}{2}}=Y
$$


S. Zhang et al.: Plastic Effect on the Sliding Inception

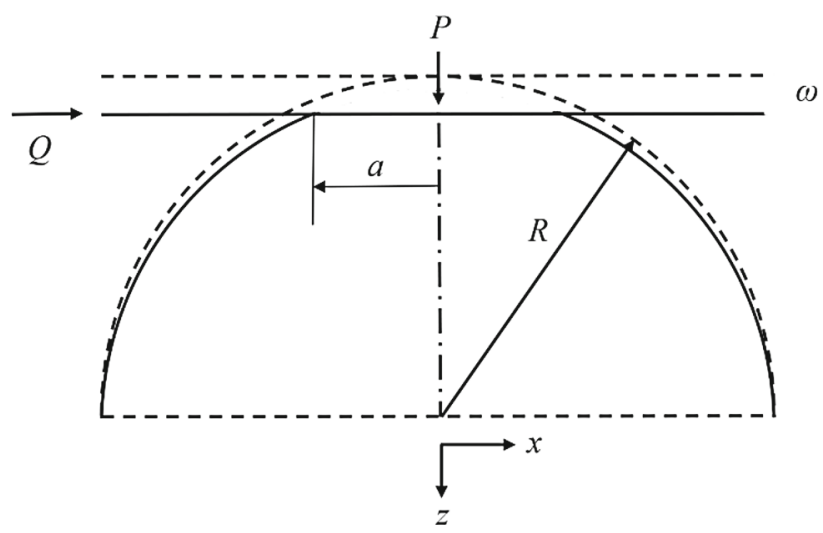

Fig. 1. Contact between an elastic-perfectly plastic cylinder and a rigid flat

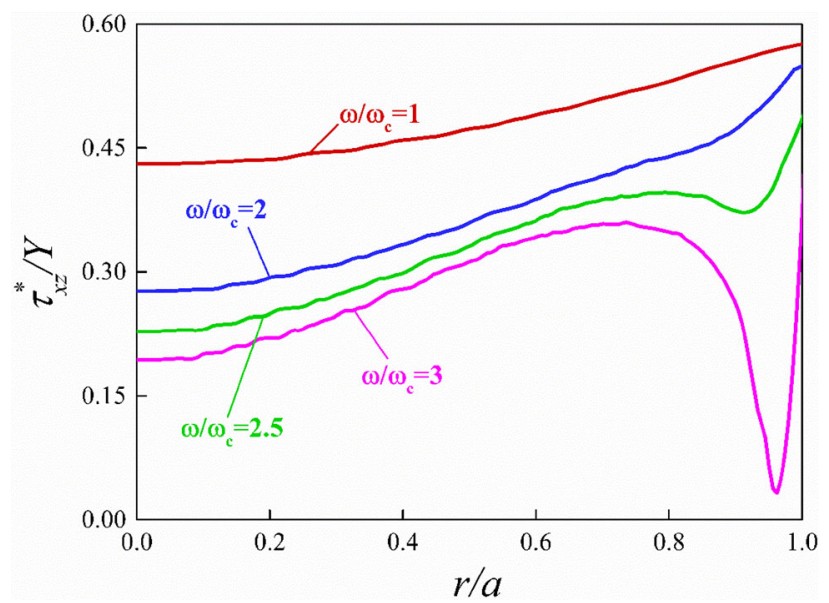

Fig. 2. Normalized maximum shear stress $\tau_{x z}^{*} / Y$ versus normalized radial coordinate $r / a$ in the contact area

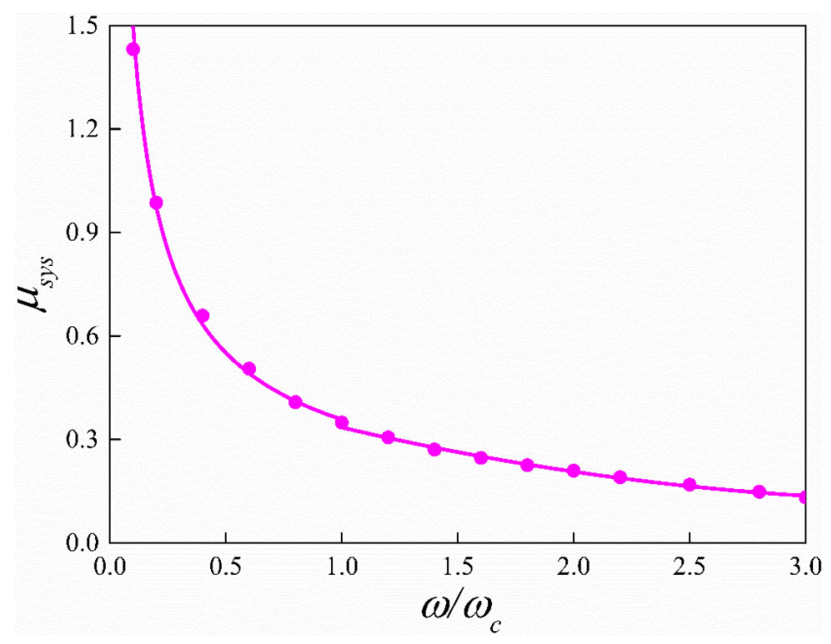

Fig. 3. System friction coefficient, $\mu_{\text {sys }}$, as a function of the interference, $\omega / \omega_{C}$ 
As the stress field on the contact surface is determined (see Appendix A), substituting $\tau_{x y}=\tau_{z y}=0$ into Eq. (1) gives the dimensionless maximum allowed local shear stress $\tau_{x z}^{*} / Y$ :

$$
\frac{\tau_{x z}^{*}}{Y}=\left\{\frac{1}{3}\left[1-\left(\frac{a}{a_{c}}\right)^{2} C^{2}(1-2 v)^{2}\left(1-\lambda^{2}\right)\right]\right\}^{\frac{1}{2}}
$$

where $\lambda=x / a, a_{c}$ is the critical contact radius, and $C$ is defined as $p_{0} / \sigma_{\text {eq }}$.

The vertical force $P$ is calculated by integrating $p(x)$ over the contact patch, while the maximum horizontal force $Q_{\max }$ is calculated by integrating $\tau_{x z}^{*}$ over the entire contact area. They have the form:

$$
P=2 \int_{0}^{a} p(x) \mathrm{d} x, \quad Q_{\max }=\int_{0}^{1} 2 a \tau_{x z}^{*} \mathrm{~d}\left(\frac{x}{a}\right)
$$

In the present work, $P_{c}$ and $\omega_{C}$ are, respectively, defined as critical vertical load and critical interference, marking the transition from elastic regime to elastic-plastic regime. The formulas of $P_{c}, a_{c}$ and $\omega_{C}$ are shown in Appendix B. The variables $P / P_{c}$ and $a / a_{c}$ as functions of $\omega / \omega_{C}$ are obtained using the finite element method (Appendix B). In our simulation, Poisson's ratio is taken as $v=0.3$, so the corresponding parameter $C=1.794$. Hence, the normalized horizontal force $Q_{\max }$ and static friction coefficient in the elastic regime have the form:

$$
\begin{aligned}
\frac{Q_{\max }}{P_{c}} & =\frac{4}{C \pi}\left(\frac{a}{a_{c}}\right) \int_{0}^{1} \frac{\tau_{x z}^{*}}{Y} \mathrm{~d} \lambda \\
\mu_{\text {sys }} & =\frac{Q_{\max }}{P}=\frac{Q_{\max } / P_{c}}{P / P_{c}}=\frac{4}{C \pi}\left(\frac{a}{a_{c}}\right)^{-1} \int_{0}^{1} \frac{\tau_{x z}^{*}}{Y} \mathrm{~d} \lambda
\end{aligned}
$$

Based on the relationships discussed above, the expression for $\mu_{\text {sys }}$ in the elastic regime can be obtained by using Eq. (2) and Eq. (5). Its expression can be fitted using Eq. (6). The simulated data and the fitting are shown in Fig. 3.

$$
\mu_{\mathrm{sys}}=0.35\left(\frac{\omega}{\omega_{C}}\right)^{-0.5}
$$

\subsection{Semi-Analytical Solution of Friction Coefficient for Elastic-Plastic Preload}

When the vertical load $P$ is larger than its critical value $P_{c}$, analytical expression for surface stress is no longer valid because of plasticity. The local stress distributions on the surface can be obtained from the finite element simulation. Then, the local maximum shear stress $\tau_{x z}^{*}$ on the contact surface can be calculated according to Eq. (1). The subsequent application of the tangential force will not change the distribution of normal pressure induced by the preload according to the FEM simulations. The reason is that we use a deformable body against a rigid plane, which is equivalent to one contact pair with two similar deformable bodies. There is no coupling between the normal and the tangential parts.

The plastic zone expands as the vertical load increases. When $\omega / \omega_{C}$ is equal to 3 , the plastic region reaches the cylinder surface, representing the upper limit of the elastic-plastic regime (Appendix B). The allowed shear stress distribution for different $\omega / \omega_{c}$ is presented in Fig. 2. In the elastic-plastic state, the values of $\tau_{x z}^{*} / Y$ can be obtained according to Eq. (2) using the finite element method. It can be seen that the value of $\tau_{x z}^{*} / Y$ decreases with increasing $\omega / \omega_{C}$. When $\omega / \omega_{C}$ is equal to 3 , the minimum value of $\tau_{x z}^{*} / Y$ is about zero, and its location denotes the free surface. The values of $\tau_{x z}^{*} / Y$ are not monotone functions of radial coordinate in the elastic-plastic regime because a crescent plastic region begins to expand beneath the contact surface. The values of $\tau_{x z}^{*} / Y$ can be integrated to obtain $Q_{\max } / P_{c}$ by using Eq. (4).

Both $a / a_{c}$ and $P / P_{c}$ are functions of $\omega / \omega_{C}$. The corresponding relations can be determined according to the finite element solution (Appendix B). Hence, the friction coefficient of the system can be expressed in the form: 

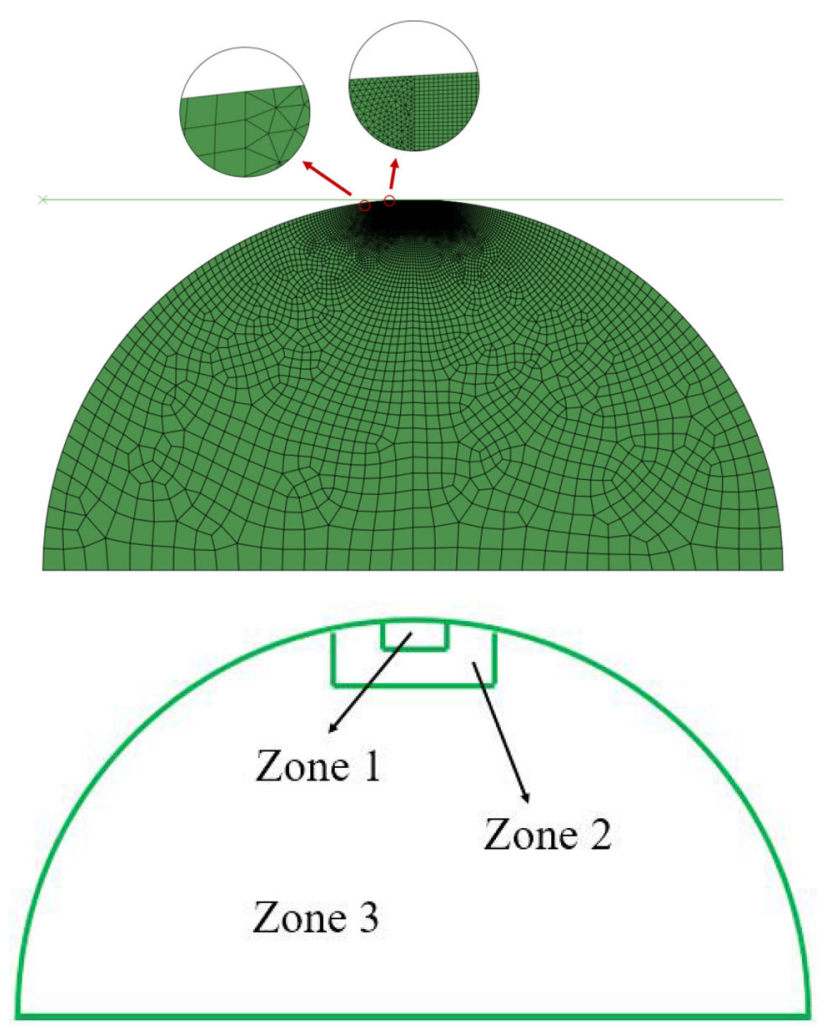

Fig. 4. Meshing schemes of an elastic-perfectly plastic cylinder in contact with a rigid flat

$$
\mu_{\mathrm{sys}}=\frac{Q_{\max }}{P}=\frac{Q_{\max } / P_{c}}{P / P_{c}}=\frac{3.782}{C \pi}\left(\frac{\omega}{\omega_{C}}\right)^{-0.287} \int_{0}^{1} \frac{\tau_{x z}^{*}}{Y} \mathrm{~d} \lambda
$$

The friction coefficient of the system can be calculated, and by proper fitting, the empirical expression for $\mu_{\text {sys }}$ can be obtained:

$$
\mu_{\text {sys }}=-0.016\left(\frac{\omega}{\omega_{C}}\right)^{3}+0.124\left(\frac{\omega}{\omega_{C}}\right)^{2}-0.382 \frac{\omega}{\omega_{C}}+0.606
$$

The value of $\mu_{\text {sys }}$ is calculated according to Eq. (5) and Eq. (8). The relationship between $\mu_{\text {sys }}$ and the normalized interference $\omega / \omega_{C}$ in the elastic and elastic-plastic regimes is shown in Fig. 3. The $\mu_{\text {sys }}$ decreases with increasing $\omega / \omega_{C}$. The empirical expression for $\mu_{\text {sys }}$ in the elastic and elastic-plastic regimes (that is, $\omega / \omega_{C}$ ranges from 0 to 3 ) is:

$$
\begin{array}{ll}
\mu_{\text {sys }}=0.35\left(\frac{\omega}{\omega_{C}}\right)^{-0.5} & \text { for } 0<\frac{\omega}{\omega_{C}}<1 \\
\mu_{\text {sys }}=-0.016\left(\frac{\omega}{\omega_{C}}\right)^{3}+0.124\left(\frac{\omega}{\omega_{C}}\right)^{2}-0.382 \frac{\omega}{\omega_{C}}+0.606 & \text { for } 1 \leq \frac{\omega}{\omega_{C}} \leq 3
\end{array}
$$

\section{A Numerical Model of Sliding Inception from Full-Slip to Full-Stick Conditions}

The semi-analytical solution in Sect. 2 is only valid for full-stick condition. In a realistic material interface, the finite slip governed by interfacial strength exists. The sliding initiation can be caused by full slip, partial slip or full stick. The factor determining the transition from partial-slip condition to full-stick condition is still unclear, which, however, cannot be obtained from the semi-analytical solution given in the previous section, either. Thus, in this section, a friction model with contact stiffness criterion is presented to investigate this effect. The transition from full-slip condition to fullstick condition is studied. 

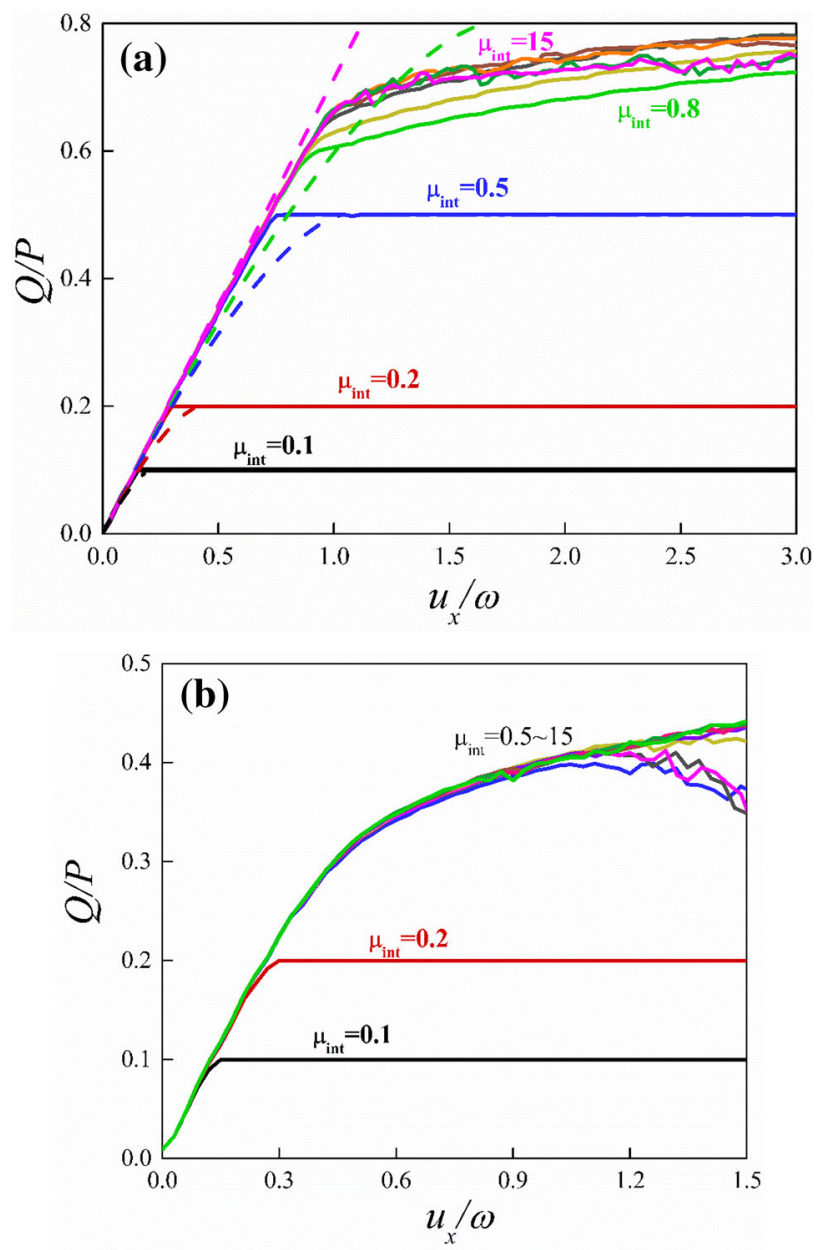

Fig. 5. Typical response of normalized friction force versus normalized horizontal loading displacement. a $\omega^{*}=0.5$. $\mathbf{b}$ $\omega^{*}=50$

The combined vertical and horizontal loads of a rigid flat on a cylinder are simulated by using the finite element package ABAQUS. The cylinder radius $R$ is $5 \mathrm{~mm}$, and the length of the rigid flat is 10 $\mathrm{mm}([17,18]$ and $[19,20])$. The finite element model is divided into three zones with different mesh densities. Zone 1 is $0.011 \% R$ distance from the cylinder tip (see Fig. 4), whose mesh density is $0.04 \% R$, consisting of four-node bilinear plane strain quadrilateral elements. Zone 2 is $0.11 \% R$ distance from the cylinder tip, whose mesh density is $0.06 \% R$, consisting of three-node linear plane strain triangle elements. Mesh transition is used in Zone 3 which is far away from the contact region. Denser mesh in Zone $1(0.005 \% R, 0.0025 \% R)$ is also checked for mesh sensitivity, which shows similar results.

The material of cylinder is modeled as elastic-perfectly plastic, and the Poisson's ratio is taken as 0.3 , which is similar to the study of Jackson et al. [21, 22]. Three sets of material yield strength $\left(\sigma_{y} / E=0.25 \%, 0.5 \%, 0.75 \%\right)$ are studied to check the material dependence. Results reveal that these three cases show similar normal and tangential responses. The dimensionless critical interference $\omega^{*}=$ $\omega / \omega_{C}$ is used to determine the preload state before sliding.

The cylinder is under plane strain condition and the bottom of the cylinder is fixed in all directions. Loads are applied in two steps: vertical load first, followed by horizontal load. Both the vertical and horizontal loads are applied under displacement control. The interfacial friction coefficient $\mu_{\text {int }}$ is served as an independently controlled parameter to study the failure mechanism of shear behavior. The value of $\mu_{\text {int }}$, ranging from 0.1 to $10^{4}$, is set to simulate the interfacial condition from partial slip to full stick. Results show that there is no big difference between the cases with $\mu_{\text {int }}=15$ and $\mu_{\text {int }}=10^{4}$. Therefore, $\mu_{\mathrm{int}}=15$ is used to present the full-stick condition. 

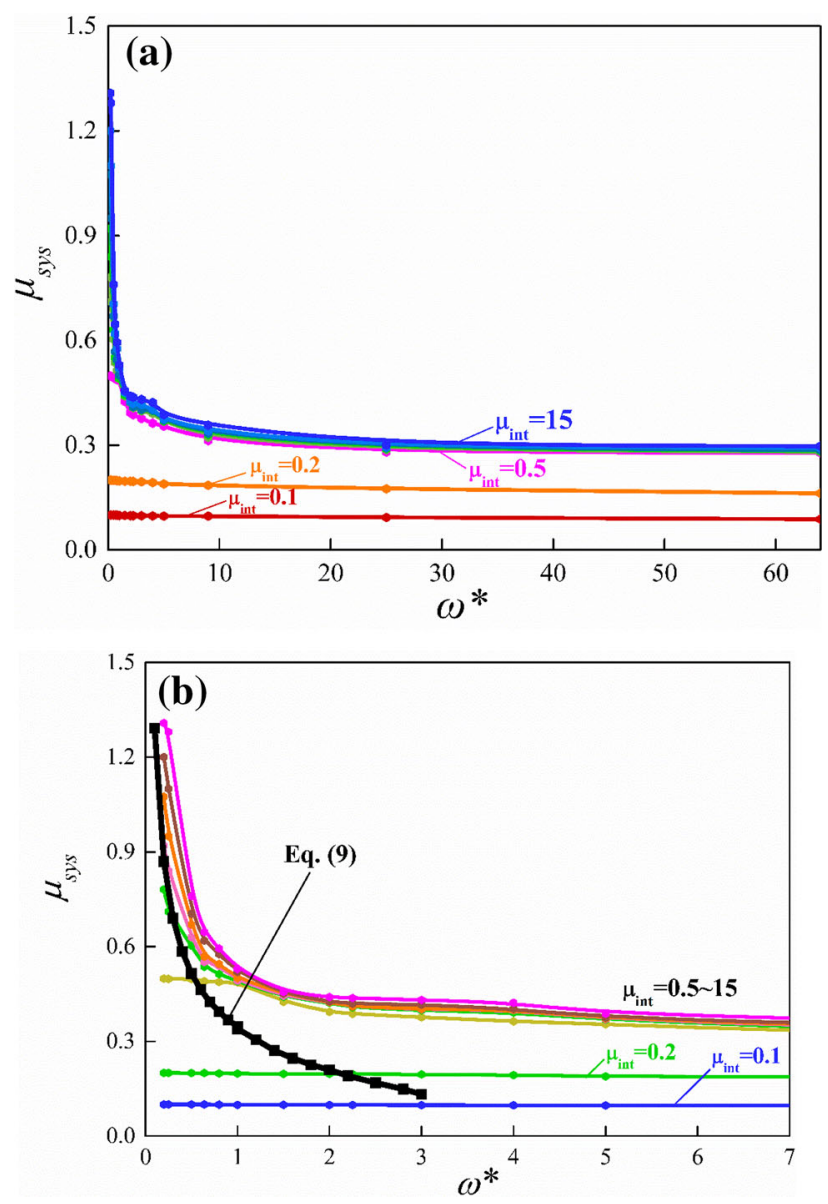

Fig. 6. Effect of plasticity on friction behavior. a $\omega^{*} \leq 64$. $\mathbf{b} \omega^{*} \leq 7$

After vertical loading, a horizontal displacement $u_{x}$ of the flat is applied. The instantaneous horizontal force $Q$ is obtained from the $x$-component of the reaction force. The corresponding instantaneous tangential stiffness $K_{T}$ [10] of the junction is calculated as:

$$
\left(K_{T}\right)_{i}=\left(\frac{\partial Q}{\partial u_{x}}\right)_{i} \approx \frac{Q_{i}-Q_{i-1}}{\left(u_{x}\right)_{i}-\left(u_{x}\right)_{i-1}}
$$

where $i$ is the increment of consecutive tangential displacement. A criterion for sliding inception is set in the form:

$$
\frac{\left(K_{T}\right)_{i}}{\left(K_{T}\right)_{0}} \leq \alpha
$$

where $\left(K_{T}\right)_{0}$ is the initial tangential stiffness of the joint corresponding to the first horizontal loading step, and $\alpha$ is a small pre-defined number. The value of 0.1 is selected as in literature [10], since a lower value of $\alpha$ negligibly increases $Q_{\max }$, but requires a much higher cost of computing time.

From the macroscale experiment, the maximum static friction force is determined by the drop of tangential force on the tangential force-displacement curve. The systematic instability denotes the sliding inception, where the macroscale static friction coefficient can be measured. In our study, we are aiming to investigate the effect of interaction between the local Amontons' law and material plasticity on the systematic instability. There exist two extreme cases: (1) the instability of purely elastic interface is only determined by the interfacial slip with Amontons' law, as stated by the reviewer, and (2) the instability of full-stick interface will not appear in purely elastic contact and will be dominated by yielding for plastic case. By plotting the system energy as a function of horizontal displacement, we 

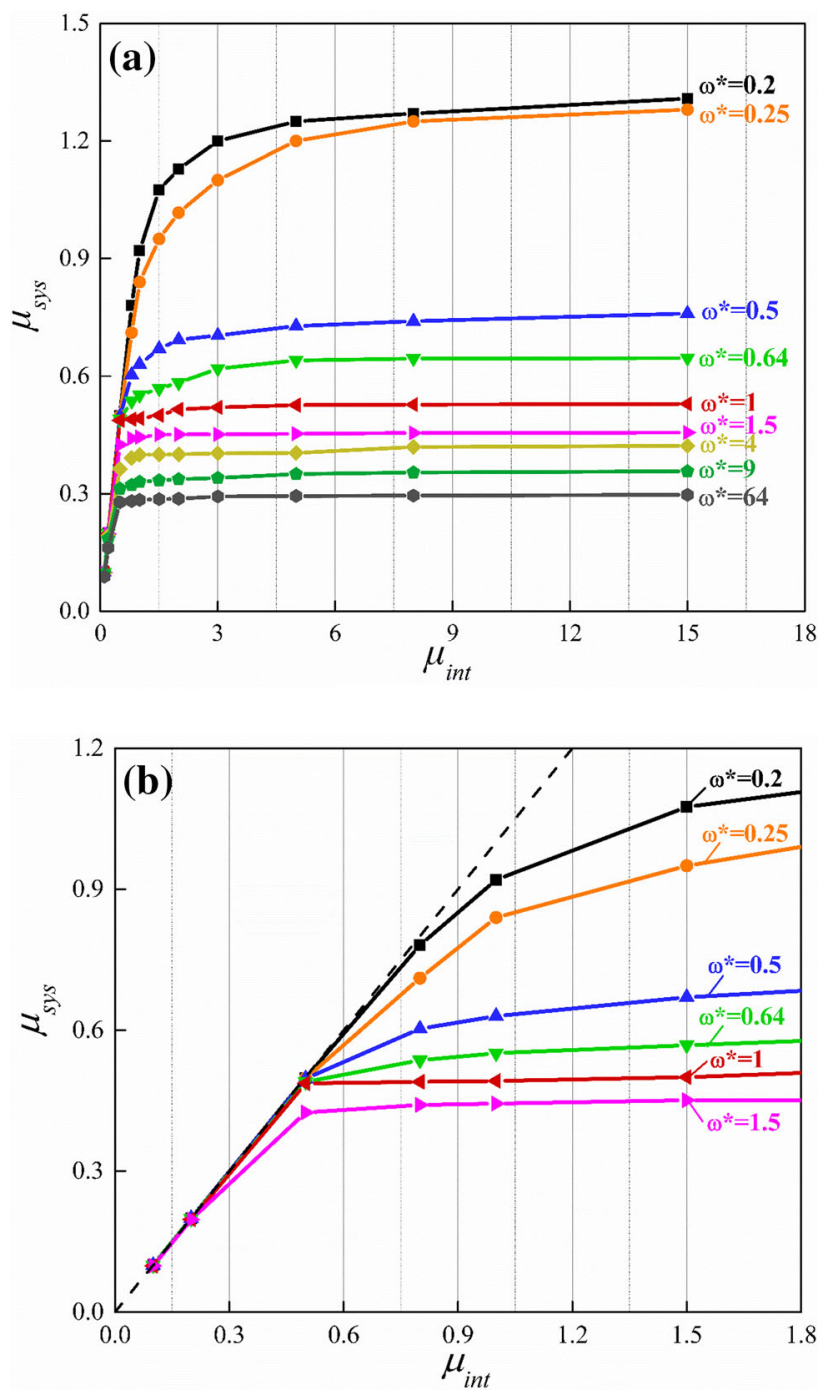

Fig. 7. Effect of interface on overall friction behavior, which shows that the friction coefficient of full-stick interface converges at a stable value. a $\mu_{\text {int }}<15$. b $\mu_{\text {int }} \leq 1.5$

conclude that the system energy reaches a minimum value as the tangential stiffness approaches zero. The criterion is based on the energy of the system, so it is irrelevant to whether the contact interface is full stick or full slip. As a result, the local Amontons' law is not able to uniquely determine the friction coefficient of the system $\mu_{\text {sys }}$.

\subsection{Friction Response}

\section{Results and Discussion}

Results from the finite element simulations are shown in this section. Figure 5 shows typical friction forces as a function of sliding distance at two different levels of interference: elastic interference in Fig. 5a and plastic interference in Fig. 5b. Horizontal loading starts with an elastic response, which shows almost a constant stiffness. Then, the horizontal force reaches a plateau (the full-slip regime) right after the linear regime when the value of interfacial friction $\mu_{\text {int }}$ is low, for example, $0.1,0.2$ and 0.5. As the sliding barrier increases, the tangential response shows full-stick feature as $\mu_{\text {int }}$ is larger than 0.8 . The horizontal load $Q_{\max }$ at sliding inception can be determined by the instability criterion as in Eq. (10). It is the static friction force from which the static friction coefficient can be calculated.

From the semi-analytical solution of Eq. (9) for full-stick case, the theoretical value of $\mu_{\text {sys }}$ decreases with the increase in interference $\omega^{*}$, as shown in Fig. 6b. Figure 6 also shows the variation of $\mu_{\text {sys }}$ from 

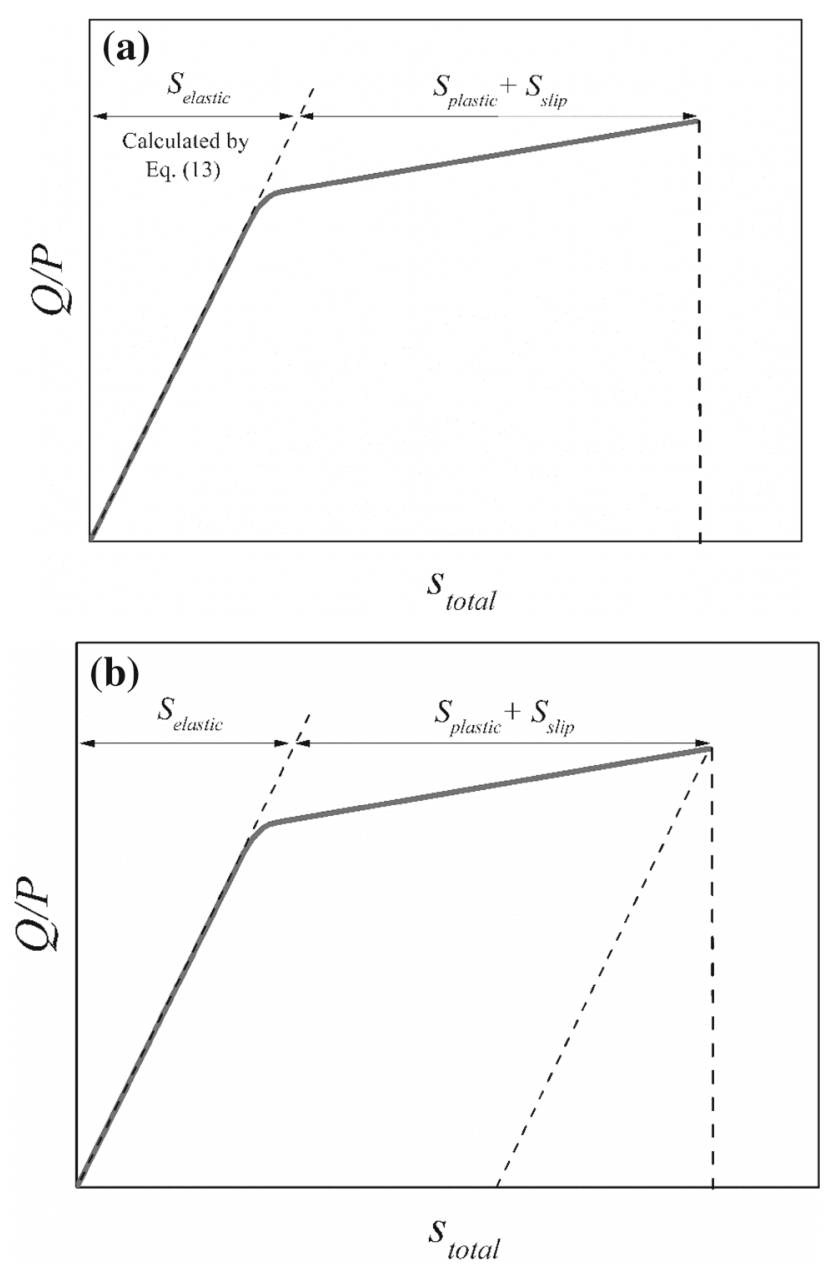

Fig. 8. Diagram of force-displacement relationship. a Elastic regime. b Plastic regime

the finite element simulation under the finite-slip condition. It should be noted that $\mu_{\text {sys }}$ converges to an up-limit line when $\mu_{\text {int }}$ is large than 0.8 , corresponding to the full-stick condition. Reminding that the semi-analytical solution of Eq. (9) is only valid for the full-stick case, Eq. (9) shows a similar trend, but always underestimates the friction coefficient compared with the FEM. The deviation comes from the assumption that in the theoretical model the contact stress field does not change with the applied horizontal load. This assumption is not the case by checking the stress field from the FEM results. With the increase in initial plasticity, the value of $\mu_{\text {sys }}$ converges to 0.3 , which agrees with Archard's assumption and the BKE model [10].

For ideal elastic interfaces, the system friction coefficient $\mu_{\text {sys }}$ is equal to the local friction coefficient, as shown by the dashed line in Fig. 7b. However, the value of $\mu_{\text {sys }}$ deviates from the elastic trend when taking the plastic effect into account. As the difficulty in interfacial slip increases, the plasticity contribution goes up in such a way that $\mu_{\text {sys }}$ reaches a plateau and maintains in a steady state, as shown in Fig. 7a. In the regime where $\mu_{\text {int }}$ ranges from 0.1 to 1.5, the friction transition from full slip to plastic yielding is more explicit, as shown in Fig. $7 \mathrm{~b}$.

\subsection{Plastic Effect}

In this section, we discuss the plastic effect on tangential shear. The total shear relative displacement $s_{\text {total }}$ of contact pair is decomposed into three parts: the elastic deforming displacement $s_{\text {elastic }}$, the plastic deforming displacement $s_{\text {plastic }}$ and the interfacial slip part $s_{\text {slip }}$ :

$$
s_{\text {total }}=s_{\text {elastic }}+s_{\text {plastic }}+s_{\text {slip }}
$$



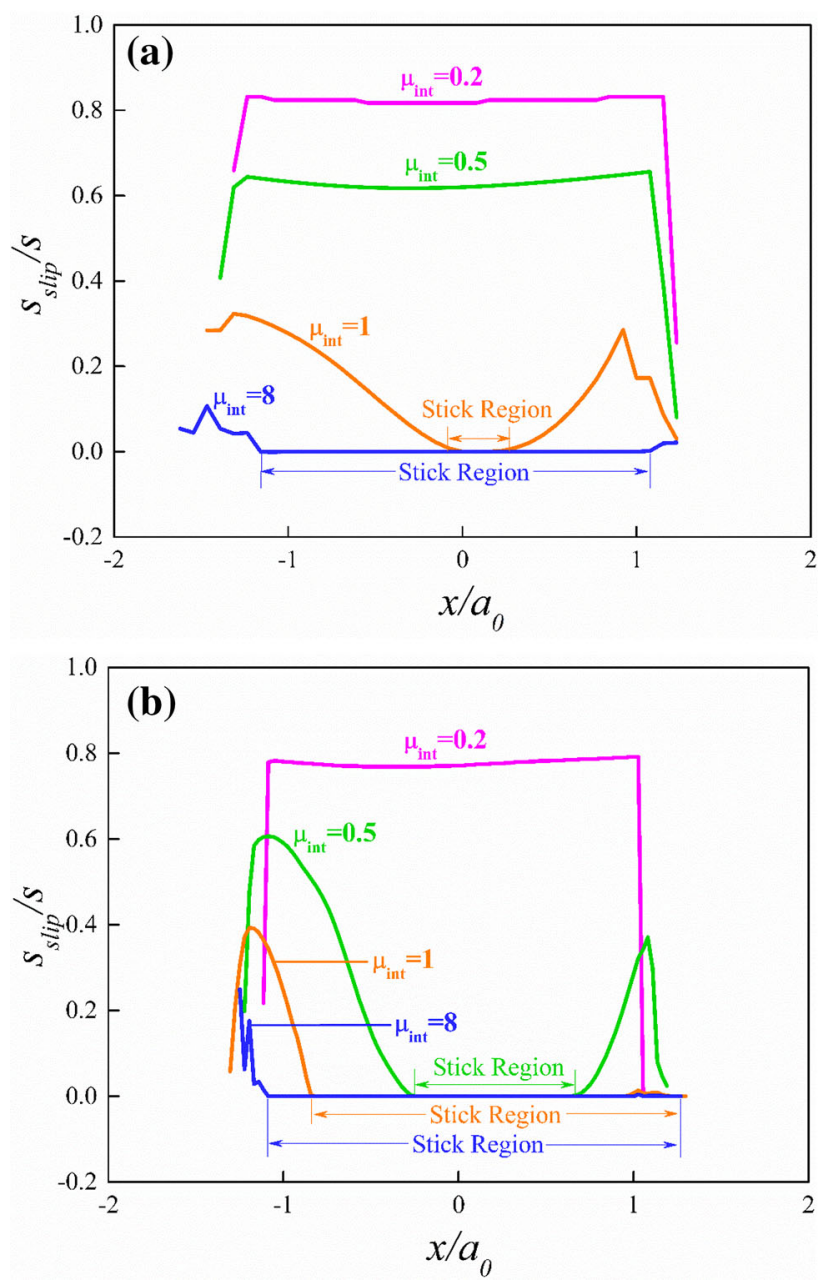

Fig. 9. Slip distributions along the contact patch. $\mathbf{a} \omega^{*}=0.2$. $\mathbf{b} \omega^{*}=1.0$

When the contact is in the elastic regime, the displacement can be calculated using the analytical method. As the cylinder is considered as infinite, we assume that the contact area is an ellipse with semi-axes $a$ and $b$, where $b>>a$. The tangential displacement can be calculated according to Vermeulen and Johnson [23]:

$$
s_{\text {elastic }}=\frac{\mu p_{0} a}{2 G}\left[1-\left(1-\frac{Q}{\mu P}\right)^{\frac{2}{3}}\right] \Gamma\left(\frac{a}{b}\right)
$$

where $\Gamma$ is a function of the complete elliptic integrals according to Jaknke and Emde [24]. We assume that $a / b=0.01$, so that the semi-axis $b$ is sufficiently long and the value of $s_{\text {elastic }}$ can be calculated for the value of cylindrical contact case. The analytical relationships between $Q / P$ and the displacement are shown by the dash lines in Fig. 5a. When the contact is in the plastic regime, the contribution of $s_{\text {elastic }}, s_{\text {plastic }}$ and $s_{\text {slip }}$ can be divided according to the diagram in Fig. $8 \mathrm{~b}$.

The value of $s_{\text {slip }}$ reflects the magnitude of interfacial slip. Figure 9 shows the slip distribution at the inception of sliding with different initial interferences. It can be seen that the central area (where $s_{\text {slip }}$ is zero) represents the stick region of the contact, which expands with the increase of $\mu_{\mathrm{int}}$. Comparing Fig. 9a with Fig. 9b, it can be seen that increasing initial interference will decrease the slip region, which leads to a more sticky sliding inception.

Figure $10 \mathrm{a}$ and b presents the proportions of $s_{\text {plastic }}, s_{\text {elastic }}$ and $s_{\text {slip }}$ in terms of $\mu_{\text {int }}$ and $\omega^{*}$. It can be seen that the proportion of $s_{\text {plastic }}$ increases with the increase in interfacial condition $\mu_{\text {int }}$ and 

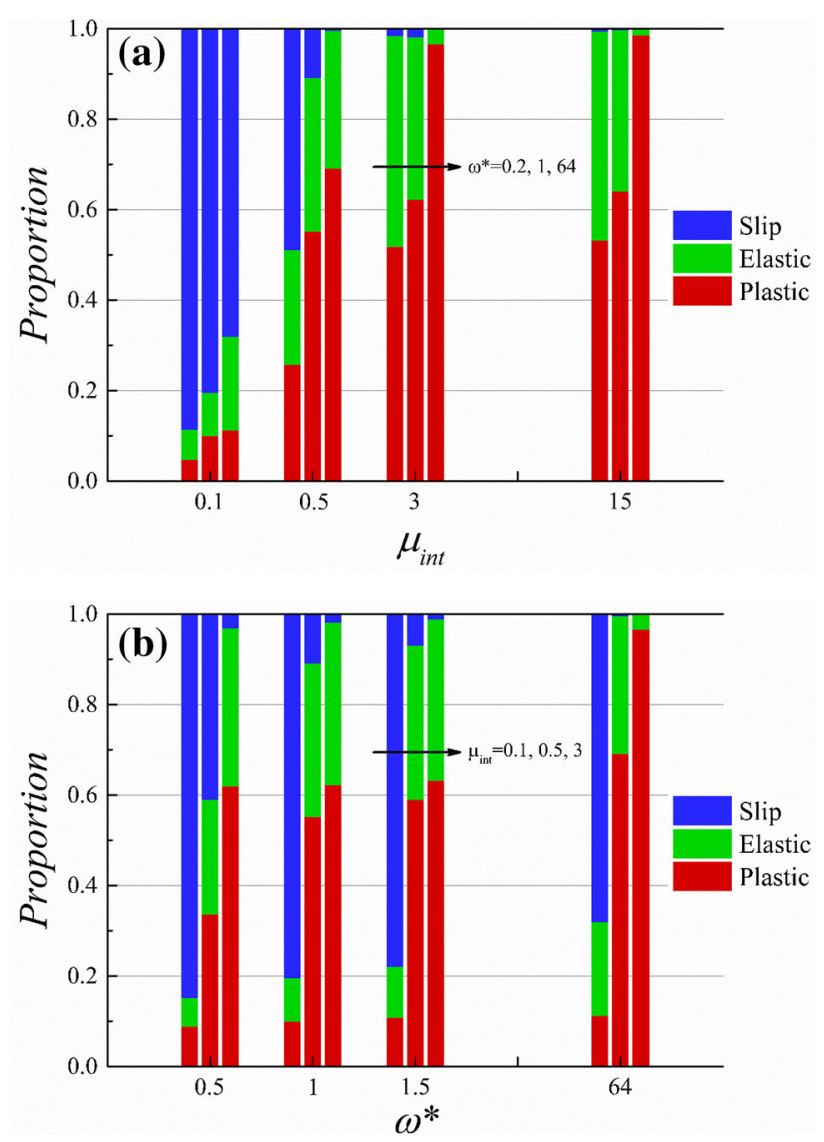

Fig. 10. Proportions of plastic deformation, elastic deformation and slip under different interfacial conditions

initial $\omega^{*}$. Figure 10a shows that, in most practical cases, as $\mu_{\text {int }}$ is smaller than 0.5 , the percentage of $s_{\text {plastic }}$ is less than $10 \%$, which means interfacial slip dominates the sliding. In the cases that $\mu_{\text {int }}$ is larger than 10, the proportion of $s_{\text {slip }}$ is close to 0 and most of the deformation is plastic. It should be noted that even with a large value of initial $\omega^{*}, s_{\text {slip }}$ can be a dominant part if the interfacial friction $\mu_{\text {int }}$ is very low.

Figure 11a shows the contribution of plastic deformation $s_{\text {plastic }}$ under different interfacial conditions and magnitudes of normal interference. When $\mu_{\text {int }}$ is smaller than 0.3 , the plastic displacement ratio $s_{\text {plastic }} / s_{\text {total }}$ is less than $30 \%$, so interfacial slip dominates the sliding in this region. When $\mu_{\text {int }}$ is larger than 1 , the ratio $s_{\text {plastic }} / s_{\text {total }}$ continues to increase until reaching $100 \%$; therefore, in this region, material yielding gradually dominates the sliding. During the transition stage $\left(0.2<\mu_{\text {int }}<0.8\right)$, the change of plastic displacement ratio $s_{\text {plastic }} / s_{\text {total }}$ represents a transition from interfacial slip dominant to plasticity dominant.

Figure 11b shows the contribution of plastic deformation $s_{\text {plastic }}$ in terms of initial $\omega^{*}$. It can be seen that when $\mu_{\text {int }}$ is relatively small $\left(\mu_{\text {int }}=0.1,0.2\right.$, full slip), the preload state $\omega^{*}$ has little influence on the slip profile. When $\mu_{\text {int }}$ is relatively large $\left(\mu_{\text {int }}>0.8\right.$, full stick), the corresponding plastic displacement ratio shows the same changing trend. However, during the transition stage $\left(0.2<\mu_{\text {int }}<0.8\right)$, the ratio $s_{\text {plastic }} / s_{\text {total }}$ changes differently, representing the transition from slip to stick.

\subsection{Comparison with Other Models}

A semi-analytical solution is useful not only in spherical contact, but also in line/cylindrical contact; however, the corresponding results are completely different. We compare the relationship between the maximum horizontal load $Q_{\max } / P_{c}$ and the contact load $P / P_{c}$, as shown in Fig. 12. Kogut and Etsion [4] concluded that $Q_{\max } / P_{c}$ is 0 when $P / P_{c}$ is larger than 14 . They underestimated the capacity of horizontal load during the plastic stage, because from our work, $Q_{\max } / P_{c}$ is a monotonic function 

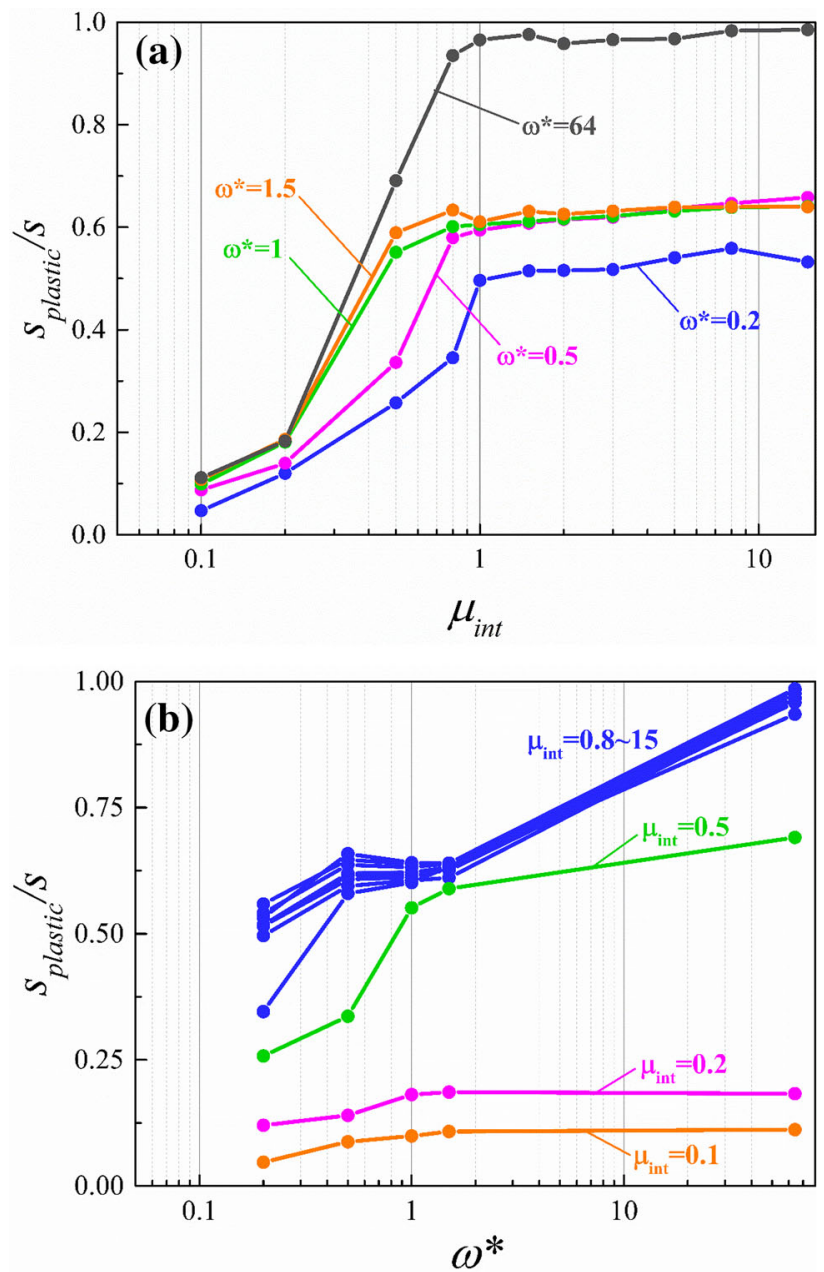

Fig. 11. Contribution of slip caused by plasticity under different interfacial conditions

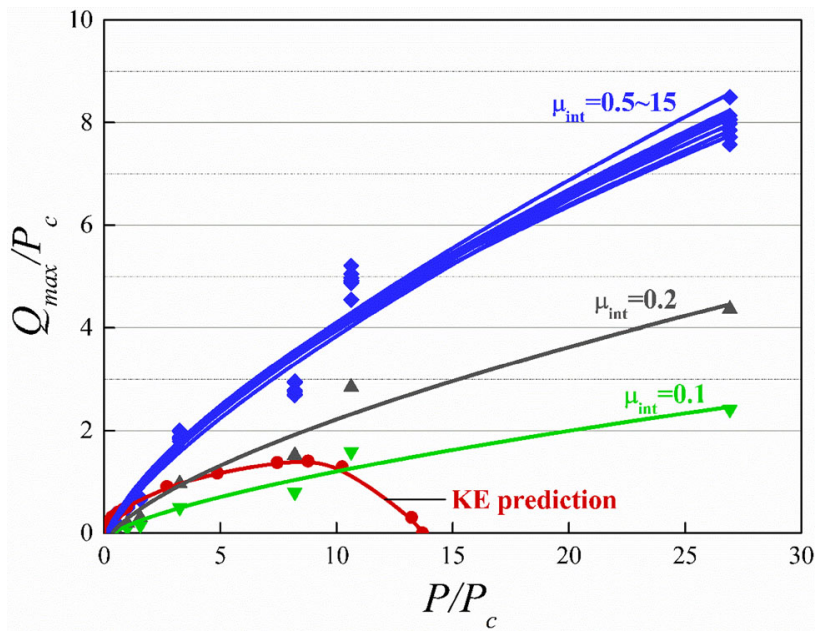

Fig. 12. Maximum horizontal load as a function of contact vertical force 

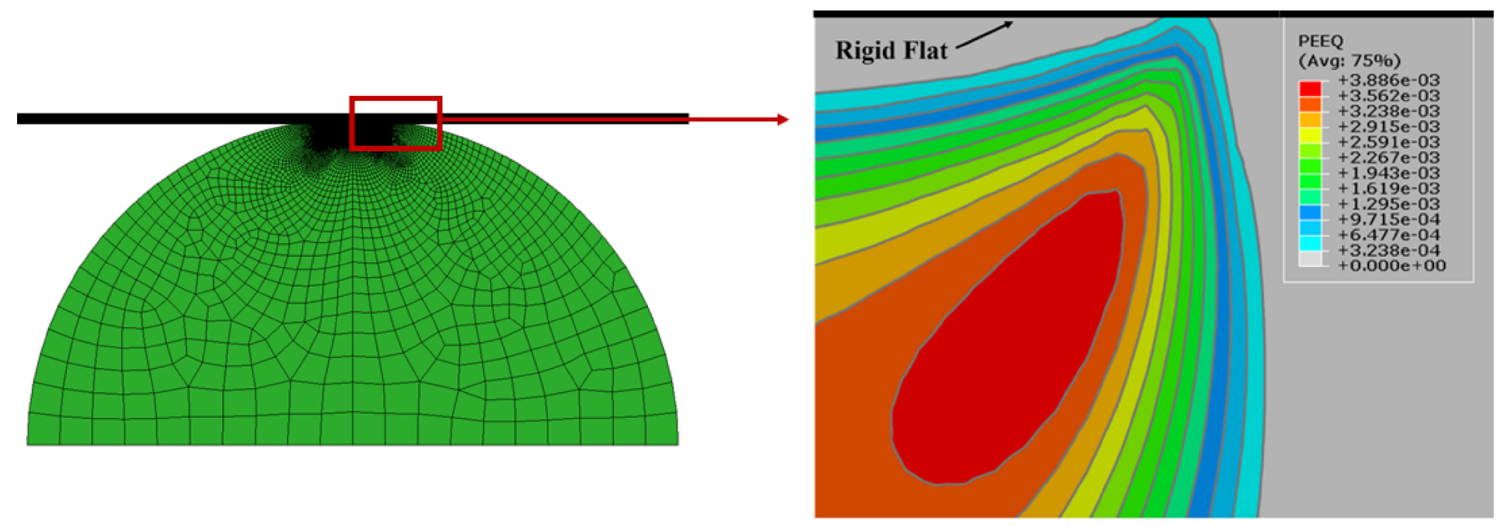

Fig. 13. Equivalent plastic strain distribution $\left(\omega^{*}=3\right)$

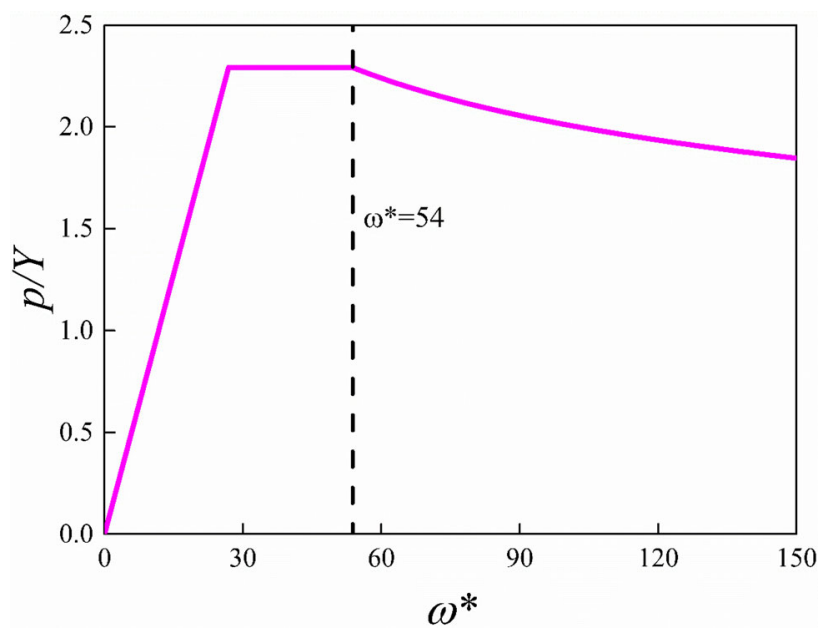

Fig. 14. Normalized mean contact pressure, $p / Y$, versus the dimensionless interference $\omega^{*}$, which shows that $\omega^{*}=54$ is the beginning of fully plastic regime

of $P / P_{c}$, and its value can never be zero when $P / P_{c}$ is larger than 14 , as shown in Fig. 11 . The relationship between $Q_{\max } / P_{c}$ and $P / P_{c}$ is affected by the value of interfacial friction coefficient $\mu_{\text {int }}$, as $\mu_{\text {int }}$ changes from 0.5 to full-stick condition and $Q_{\max } / P$ coincides with one line with a slope equal to about 0.3 .

\section{Conclusion}

1. A semi-analytical model for full-stick case, as in Eq. (9), is able to predict the friction coefficient with the change of interference.

2. A friction model with contact stiffness criterion is proposed. It is implemented in the finite element simulations to study both material plasticity and local slip effects on the friction map. When $\mu_{\text {int }}$ is smaller than 0.3 , interfacial slip dominates the sliding. When $\mu_{\text {int }}$ is larger than 0.8 , material yielding dominates the sliding. In the interfacial system with $0.3<\mu_{\text {int }}<0.8$, a transition mechanism should be considered by comparing the contributions from interfacial strength and material plasticity.

3. Plasticity saturation appears as interference $\omega^{*}$ is larger than 3 . The friction coefficient of full-stick interface converges at a stable value of 0.3 .

Acknowledgements. This work was supported by the National Natural Science Foundation of China (Grant Nos. 11772334, 11432014, 11672301), the Strategic Priority Research Program of the Chinese Academy of Sciences (Grant No. XDB22040501) and the State Key Laboratory of Structural Analysis for Industrial Equipment (DUT Grant No. GZ15116). 


\section{Appendix A: The Stress Field Under Vertical Load}

According to the coordinate system in Fig. 1, the stress field on the axis of symmetry is presented as $([25,26])$ :

$$
\frac{\sigma_{x}}{p_{0}}=-\left(\frac{1+2 \varsigma^{2}}{\sqrt{1+\varsigma^{2}}}-2 \varsigma\right) ; \quad \frac{\sigma_{z}}{p_{0}}=-\frac{1}{\sqrt{1+\varsigma^{2}}} ; \quad \frac{\sigma_{y}}{p_{0}}=2 v\left(\varsigma-\sqrt{1+\varsigma^{2}}\right) ; \quad \frac{\tau_{x y}}{p_{0}}=\varsigma-\frac{\varsigma^{2}}{\sqrt{1-\varsigma^{2}}}
$$

where $\varsigma=z / a$. As a result, the equivalent stress in the axis of symmetry has the form:

$$
\begin{aligned}
\frac{\sigma_{\mathrm{eq}}}{p_{0}}= & f\left(\frac{z}{a}\right)=\left(4 \varsigma^{2}-\frac{8 \varsigma^{3}}{\sqrt{1+\varsigma^{2}}}+\frac{4 \varsigma^{4}+1}{1+\varsigma^{2}}+\frac{4 v \varsigma}{\sqrt{1+\varsigma^{2}}}-4 v+8 v^{2} \varsigma^{2}+4 v^{2}\right. \\
& -8 v^{2} \varsigma \sqrt{1+\varsigma^{2}}+\frac{4 \varsigma^{3} v}{\sqrt{1+\varsigma^{2}}}+4 \varsigma v \sqrt{1+\varsigma^{2}}-8 \varsigma^{2} v+\frac{2 \varsigma^{2}}{1+\varsigma^{2}} \\
& \left.-\frac{2 \varsigma}{\sqrt{1+\varsigma^{2}}}\right)^{1 / 2}
\end{aligned}
$$

Similar to Jackson [21], the location $\varsigma_{Y}$ with maximum von Mises stress can be obtained by using equation: $\left.\frac{\partial}{\partial \varsigma}\left(\frac{\sigma_{\text {eq }}}{p_{0}}\right)\right|_{\varsigma=\varsigma Y}=0$. Substituting $\varsigma_{Y}$ into Eq. (A2) and using $\left.\sigma_{\text {eq }}\right|_{\varsigma=\varsigma_{Y}}=Y$, the relationship between $\varsigma_{Y}$ and the Poisson's ratio $v$ can be obtained. Hence, the fitting function can be expressed by Eq. (A3). And the dimensionless parameter $C=\left(\frac{\sigma_{\mathrm{eq}}}{p_{0}}\right)^{-1}$ can also be obtained in Eq. (A3).

$$
\begin{aligned}
\varsigma_{Y} & =-2.39 v^{2}+2.32 v+0.222 \\
C & =-2.92 v^{2}+2.98 v+1.163
\end{aligned}
$$

The stress field at the surface is shown in Eq. (A4), which is used to calculate the friction coefficient in the elastic regime.

$$
\begin{aligned}
& \frac{\sigma_{x}}{p_{0}}=\frac{\sigma_{z}}{p_{0}}=-\left(1-\frac{x^{2}}{a^{2}}\right)^{\frac{1}{2}} \\
& \frac{\sigma_{y}}{p_{0}}=-2 v\left(1-\frac{x^{2}}{a^{2}}\right)^{\frac{1}{2}}
\end{aligned}
$$

\section{Appendix B: Fitting Parameters}

According to Johnson [25], the critical contact radius $a_{c}$, the critical vertical load $P_{c}$ and the critical interference $\omega_{C}$ can be determined by the following equations:

$$
\begin{aligned}
P_{c} & =\frac{\pi R(C Y)^{2}}{E^{*}} \\
a_{c} & =\frac{2 R C Y}{E^{*}} \\
\omega_{C} & =P_{c} \frac{1-v^{2}}{\pi E}\left[2 \ln \left(\frac{4 R}{a_{c}}\right)-1\right]
\end{aligned}
$$

where $\frac{1}{E^{*}}=\frac{1-v^{2}}{E}$, representing the equivalent Young's Modulus in the present work.

The contact stays elastically when the normalized interference $\omega^{*}=\omega / \omega_{C}$ is less than 1 . The contact enters the elastic-plastic region as $\omega^{*}$ is larger than 1 . The plastic zone expands, as it reaches the surface $\left(\omega^{*}=3\right)$, as shown in Fig. 13, and the indentation enters the plastic state. It is consistent with the simulation [11], in which the cylinder surface begins to yield once the interference reaches about 3 to 3.5 times $\omega^{*}$. As the vertical load increases, the mean contact pressure $p / Y$ increases until it reaches the full plastic region, from which $p / Y$ decreases. Figure 14 shows the change of $p / Y$ when the interference $\omega^{*}$ increases from 0 to 200 . The value of $p / Y$ starts to decrease at point $\omega^{*}=54$, which represents the fully plastic regime. Thus, we define that the contact is in fully plastic regime when $\omega^{*}>54$. 
The entire elasto-plastic response is divided into four regimes, which is similar to the KE model [27]. It is listed as follows:

\begin{tabular}{ll}
\hline Elastic state & $\omega^{*} \leq 1$ \\
Elastic-plastic state & $1<\omega^{*} \leq 3$ \\
Plastic state & $3<\omega^{*} \leq 54$ \\
Fully plastic state & $\omega^{*}>54$ \\
\hline
\end{tabular}

We parametrize the FEM results for contact force $P$ and contact area $A$ as the functions of interference in each regime through a power law:

$$
\frac{P}{P_{c}}=\alpha\left(\frac{\omega}{\omega_{C}}\right)^{m} ; \frac{A}{A_{c}}=\beta\left(\frac{\omega}{\omega_{C}}\right)^{n}
$$

The following table presents the fitting parameters.

\begin{tabular}{lllll}
\hline & $\alpha$ & $m$ & $\beta$ & $n$ \\
\hline $0<\frac{\omega}{\omega_{C}} \leq 1$ & 0.998 & 1.117 & 0.998 & 0.551 \\
$1<\frac{\omega}{\omega_{C}} \leq 3$ & 1.022 & 1.039 & 0.966 & 0.752 \\
$3<\frac{\omega}{\omega_{C}} \leq 54$ & 1.632 & 0.710 & 2.328 & 0.647 \\
$\frac{\omega}{\omega_{C}}>54$ & 4.861 & 0.424 & 3.735 & 0.521 \\
\hline
\end{tabular}

\section{References}

[1] Mindlin RD. Compliance of elastic bodies in contact. ASME J Appl Mech. 1949;16:259-68.

[2] Hamilton GM. Explicit equations for the stresses beneath a sliding spherical contact. Proc Inst Mech Eng Part C Mech Eng Sci. 1983;197C:53-9.

[3] Chang WR, Etsion I, Bogy DB. Static friction coefficient model for metallic rough surfaces. ASME J Tribol. 1988;110:57-63.

[4] Kogut L, Etsion I. A semi-analytical solution for the sliding inception of a spherical contact. ASME J Appl Mech. 2003;125:499-505.

[5] Brizmer V, Kligerman Y, Etsion I. The effect of contact conditions and material properties on the elasticity terminus of a spherical contact. Int J Solids Struct. 2006;43:5736-49.

[6] Brizmer V, Zait Y, Kligerman Y, Etsion I. The effect of contact conditions and material properties on elastic-plastic spherical contact. J Mech Mater Struct. 2006;1:865-79.

[7] Zait Y, Kligerman Y, Etsion I. Unloading of an elastic-plastic spherical contact under stick contact condition. Int J Solids Struct. 2010;47:990-7.

[8] Etsion I, Kligerman Y, Kadin Y. Unloading of an elastic-plastic loaded spherical contact. Int J Solids Struct. 2005;42:3716-29.

[9] Ronen S, Goltsberg R, Etsion I. A comparison of stick and slip contact conditions for a coated sphere compressed by a rigid flat. Friction. 2017;5:326-38.

[10] Brizmer V, Kligerman Y, Etsion I. Elastic-plastic spherical contact under combined normal and tangential loading in full stick. Tribol Lett. 2007;25:61-70.

[11] $\mathrm{Wu} \mathrm{A}$, Shi X. Numerical investigation of adhesive wear and static friction based on the ductile fracture of junction. ASME J Appl Mech. 2013;80(4):041032.

[12] Shi X, Wu A, Zhu CM, Qu SX. Effects of load configuration on partial slip contact between an elasticplastic sphere and a rigid flat. Tribol Int. 2013;61:120-8.

[13] Wu A, Shi X, Polycarpou AA. An elastic-plastic spherical contact model under combined normal and tangential loading. ASME J Appl Mech. 2012;79(5):051001.

[14] Shi X. On slip inception and static friction for smooth dry contact. ASME J Appl Mech. 2014;81(12):121005.

[15] Bhushan B. Contact mechanics of rough surfaces in tribology: multiple asperity contact. Tribol Lett. 1998;4:1-35.

[16] Hodge PG. Plastic analysis of structures. New York: McGraw-Hill Book Company; 1959. 
[17] Owen DRJ, Hinton E. Finite elements in plasticity: theory and practice. Swansea: Pineridge Press LTD.; 1980.

[18] Liu G, Zhu J, Yu L, Wang QJ. Elasto-plastic contact of rough surfaces. Tribol Trans. 2001;44:437-43.

[19] Lin LP, Lin JF. A new method for elastic-plastic contact analysis of a deformable sphere and a rigid flat. ASME J Tribol. 2006;128:221-9.

[20] Zhao B, Zhang S, Wang QF, Zhang Q, Wang P. Loading and unloading of a power-law hardening spherical contact under stick contact condition. Int J Mech Sci. 2015;94-95:20-6.

[21] Jackson RL, Green I. A finite element study of elastoplastic hemispherical contact against a rigid flat. ASME J Tribol. 2005;127:343-54.

[22] Shi X, Zou Y, Fang H. Numerical investigation of the three-dimensional elastic-plastic sloped contact between two hemispheric asperities. ASME J Appl Mech. 2016;83(10):101004.

[23] Vermeulen PJ, Johnson KL. Contact of nonspherical elastic bodies transmitting tangential forces. ASME J Appl Mech. 1964;31:338-40.

[24] Jahnke E, Emde F. Tables of functions with formulae and curves. New York: Dover Publications; 1945.

[25] Johnson KL. Contact mechanics. Cambridge: Cambridge University Press; 1985.

[26] Tian H, Saka N. Finite element analysis of an elastic-plastic two-layer half-space: sliding contact. Wear. 1991;148:261-85.

[27] Kogut L, Etsion I. Elastic-plastic contact analysis of a sphere and a rigid flat. ASME J Appl Mech. 2002;69:657-62. 